See discussions, stats, and author profiles for this publication at: https://www.researchgate.net/publication/337727389

\title{
Chemical analysis of a lunar meteorite by laser ablation mass spectrometry
}

Article in Planetary and Space Science · December 2019

DOI: 10.1016/j.pss.2019.104816

13 authors, including:

Samira Frey

École Polytechnique Fédérale de Lausanne

3 PUBLICATIONS 10 CITATIONS

SEE PROFILE

Maike Brigitte Neuland

Institutet för rymdfysik

36 PUBLICATIONS 488 CITATIONS

ค7 Marek Tulej

Universität Bern

141 PUBLICATIONS 1,554 CITATIONS

SEE PROFILE

SEE PROFILE

Crust-mantle evolution of the Earth View project

Multireference Work on "Interfacial Chemistry: Surface Science and Electrochemistry" View project 


\title{
Chemical analysis of a lunar meteorite by laser ablation mass spectrometry
}

\author{
Samira Frey ${ }^{\mathrm{a}, *}$, Reto Wiesendanger ${ }^{\mathrm{a}}$, Marek Tulej ${ }^{\mathrm{a}}$, Maike Neuland ${ }^{\mathrm{a}}$, Andreas Riedo ${ }^{\mathrm{b}}$, \\ Valentine Grimaudo ${ }^{c}$, Pavel Moreno-García ${ }^{c}$, Alena Cedeño López ${ }^{c}$, Miklós Mohos ${ }^{c}$, \\ Beda Hofmann $^{\mathrm{d}}$, Klaus Mezger ${ }^{\mathrm{e}}$, Peter Broekmann ${ }^{\mathrm{c}}$, Peter Wurz ${ }^{\mathrm{a}}$ \\ ${ }^{a}$ Space Research and Planetary Sciences, Physics Institute, University of Bern, Sidlerstrasse 5, 3012, Bern, Switzerland \\ ${ }^{\mathrm{b}}$ Laboratory for Astrophysics, Leiden Observatory, Leiden University, Niels Bohrweg 2, NL 2333, CA, Leiden, the Netherlands \\ ${ }^{c}$ Department of Chemistry and Biochemistry, Interfacial Electrochemistry Group, University of Bern, Freiestrasse 3, 3012, Bern, Switzerland \\ d Naturhistorisches Museum Bern, Bernastrasse 15, 3005, Bern, Switzerland \\ ${ }^{\mathrm{e}}$ Institut für Geologie, Baltzertrasse 1+3, 3012, Bern, Switzerland
}

\section{Introduction}

In-situ chemical analyses of the surfaces of planets, their moons, asteroids and comets conducted during space missions are an important step towards a better understanding of the origin and evolution of our planetary system. Measuring isotope and element abundances of planetary materials, including soils, rocks and individual minerals provides information on the chemical differentiation of the planet, geological evolution and weathering processes on its surface. These chemical data place critical constraints on models for the origin and evolution of planetary bodies in the solar system (Tolstikhin and Kramers, 2008). Such investigations also support the search for bio-signatures, which are of considerable interest in the research of possible past and present life (Hays, 2017; Neubeck, 2016; Tulej, 2015; Wiesendanger, 2018). The development of space instrumentation capable of sensitive in-situ measurements of elements and their isotopes is therefore of uttermost importance to cosmochemistry and planetology. Spectroscopic methods, such as gamma-ray, X-ray or neutron spectroscopy, while yielding valuable scientific data, are often limited by their inability to measure isotope ratios and by their low sensitivity to many elements. Analytical instruments employed on planetary surfaces so far include the Alpha Proton X-Ray Spectrometer (APXS) (Rieder et al., 1997, 2003) and the Laser Induced Breakdown Spectrometer (LIBS) (Maurice et al., 2012; Wiens et al., 2012), both on the surface of Mars. However, they can provide only elemental analyses with a sensitivity down to the permille range. Sample return missions bear the risk of contamination as well as being expensive, technically challenging and increasingly difficult for far away planetary objects. In addition, such missions will be able to return only small amounts of sample material that could be insufficient for many analytical methods. For these reasons, powerful in-situ instruments are needed.

Laser Ablation and ionization Time-of-flight Mass Spectrometry
(LIMS) developed into an important analytical technique for future insitu applications in space research based on the progress in laser technology and fast electronics. Miniaturized time-of-flight mass spectrometers are especially suitable for space missions because of their light and robust design and easy operation while still maintaining the capabilities of larger laboratory instruments. For example, in a study on $\mathrm{Pb}$ isotopes the results from a miniaturized LIMS and a laboratory-sized TIMS instrument were of comparable quality (Riedo et al., 2013b). The instrument used for this study is a miniature reflectron-type laser ablation time-of-flight mass spectrometer (LMS), designed and built at the University of Bern in 2003 for the lander of the BepiColombo mission to Mercury (Rohner et al., 2003). Since its initial construction, the instrument has undergone significant improvements in the laser ablation ion source, electronics and data acquisition system. The details of the design and construction of the LMS instrument can be found in previous publications (Riedo et al., 2013a, 2013b, 2017). Recently, we developed an instrument operation mode that greatly enhances the detection sensitivity for heavier elements by preventing the lighter and highly abundant elements from reaching the detector (Wiesendanger et al., 2017).

In the current study we investigated the lunar meteorite Sayh al Uhaymir 169 (SaU169) with the LMS instrument to test its ability for precise and accurate in-situ measurements for planetary research, which cannot be performed with instrumentation currently in use on planetary surfaces. SaU169 was found in 2002 in Oman and is described in more detail by Russel et al. (2003). It consists of two different lithologies. A holocrystalline, fine-grained polymict impact-melt breccia makes up around $86 \%$ of the rock volume. This part of the meteorite is extremely enriched in K, REE and P, attesting to the presence of KREEP material. The remaining $13 \%$ are shock-lithified regolith breccia.

The sample investigated in this study contains plagioclase, metallic iron, spinel, olivine, and orthopyroxene (Gnos et al., 2004). It was

\footnotetext{
* Corresponding author.

E-mail address: samira.frey@epfl.ch (S. Frey).
} 
studied with our LIMS instrument to obtain composition data that can be used to reconstruct the petrogenesis of this material. Element abundances were measured over a square area of $450 \mathrm{~mm}^{2}$. In addition, the outline of a zircon grain was mapped and the grain was analyzed for its trace element budget.

\section{Material and methods}

LMS is a miniature reflectron type time-of-flight mass spectrometer. Details of the design and operation of the instrument can be found in previous publications (Riedo et al., 2013a and b). The instrument is located inside an ultra-high vacuum chamber and operated at a background pressure of mid $10^{-7}$ mbar. The samples are placed beneath the entrance of the instrument where they can be positioned with the help of a xyz-translation stage with $2 \mu \mathrm{m}$ positioning accuracy. A Ti-sapphire laser $(\lambda=775 \mathrm{~nm}, \tau \sim 190 \mathrm{fs}$, repetition rate $\leq 1 \mathrm{kHz}$, intensity $\leq 1$ $\mathrm{mJ} /$ pulse) is used for the ablation and ionization process. The laser beam is focused on the sample via an optical system to a spot size of about 15 $\mu \mathrm{m}$ in diameter. The produced ions enter the analyzer where they are accelerated before entering the field free drift tube (mass-to-charge separation). After passing the drift tube, the ions enter the ion mirror, get reflected, pass the drift tube again before they arrive on the micro-channel plates (MCP) detection system (Riedo et al., 2017). This is the standard TOF configuration using a time-focussing ion mirror (Rohner et al., 2003). Two high speed data acquisition cards are used to record the signals generated by the detector system. The acquired spectra are then stored on a measurement computer. A high-voltage pulser, coupled with one element of the ion optical system, can be used to remove ions of a defined mass range from detection. For example, this allows for optimization of the detection of low abundance elements at high masses. The pulser generates a temporary high-voltage pulse with about $10 \mathrm{~ns}$ rise and fall times, stopping light ions from reaching the detector and therefore increasing detection sensitivity by about a factor 4 for heavier elements (Wiesendanger et al., 2017).

The sample is placed on a stainless steel sample holder and introduced into the main vacuum chamber with a sample introduction system. The distance between the entrance of the analyzer and the sample is chosen in such a way that the focal point of the laser lies just at the sample surface. The voltage settings of the ion optics are optimized to keep sufficiently high mass resolution and high ion transmission at the same time. The optimization can be done manually or with the help of a performance optimizer (Bieler et al., 2011). The laser itself can be operated in two different modes, in standard mode, applying pulses at a rate of $1 \mathrm{kHz}$, or in group mode, where a pulse train of an adjustable number of pulses are sent to the sample, resulting in the same number of recorded spectra (typically around 100) that are accumulated in one file. Several files are accumulated for each position to reduce statistical fluctuations and increase the effective signal-to-noise ratio (SNR). Typically about $60^{\prime} 000$ to $80^{\prime} 000$ spectra can be accumulated at one spot before the peak intensities disappear, due to the sample surface moving out of the laser focus point. The sequence of these spectra can be used also for depth profiling of complex materials (Grimaudo et al., 2015, 2017; Neubeck et al., 2016; Tulej et al., 2015), but this capability was not used in this study. A raster software is used to measure at several pre-defined positions in one measurement campaign automatically. Three detector channels with different gain factors are used for the data acquisition, resulting in a high dynamic range up to 8 orders of magnitude (Riedo et al., 2017). After the measurement, the data are processed with a custom-designed analysis software (Meyer et al., 2017). The element abundances resulting from these measurements and the subsequent processing are given in units of the fraction of the number of atoms of particular element to the total number of atoms ablated.

\section{Results and discussion}

\subsection{The SaU 169 sample and EDX measurements}

Three fragments of the Sayh al Uhaymir 169 meteorite were investigated. Images of the sample pieces are shown in Fig. 1a. The meteorite consists of two lithologies, an impact-melted breccia and a regolith breccia; both of them are present in the meteorite pieces used for this study. Prior to the mass spectrometric analysis, the samples were additionally investigated with Energy-Dispersive X-ray Spectroscopy (EDX) (Russ, 1984). The information obtained from the EDX analysis served as a guiding study for the spatial distribution of $\mathrm{Zr}$ on the KREEP material surface. Zirconium in lunar material appears in higher abundances only in the form of the mineral zircon $\left(\mathrm{ZrSiO}_{4}\right)$. This mineral is unique because of its chemical and physical durability. Furthermore, it is a common host for a variety of trace elements such as Hf, Y, REE as well as U, Th and radiogenic $\mathrm{Pb}$ (Gnos et al., 2004; Lin et al., 2012), which makes it especially interesting for radio-isotopic dating methods (Allègre, 2008). Other trace elements in zircon, such as Ti can be used to estimate its crystallization temperature (Watson et al., 2006; Ferry and Watson, 2007). Several zircon grains were located on the exposed sample surface

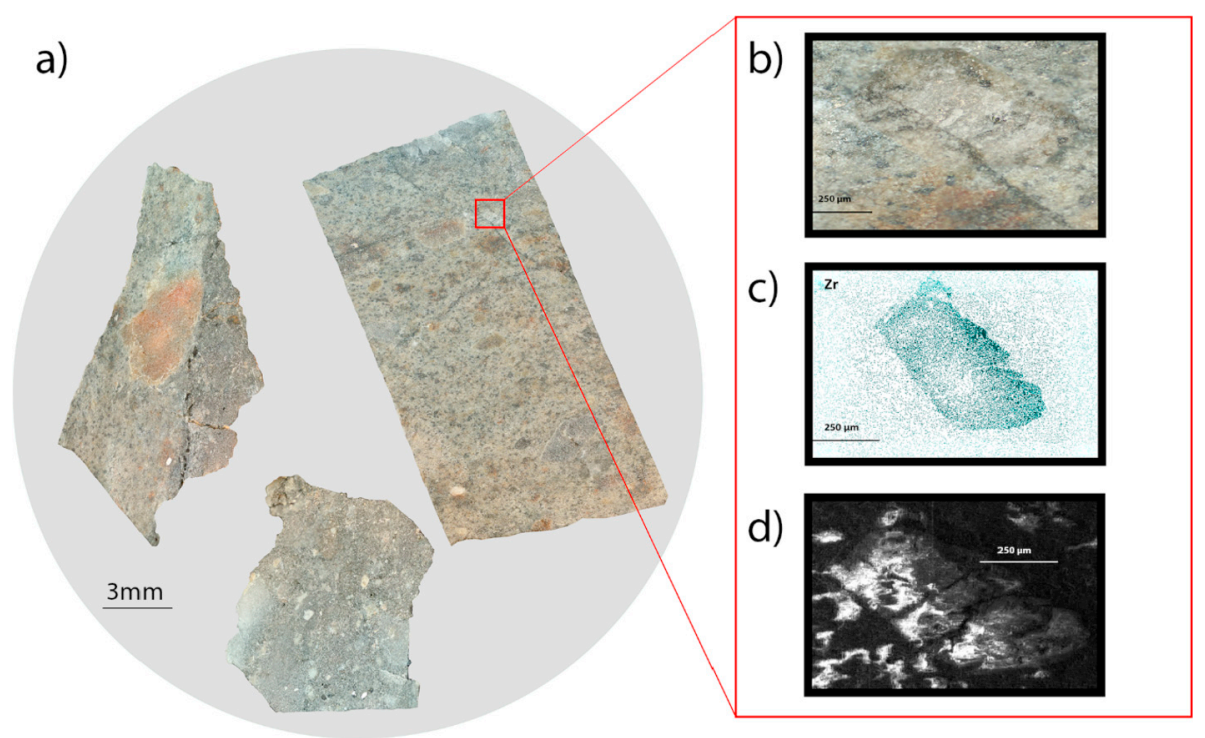

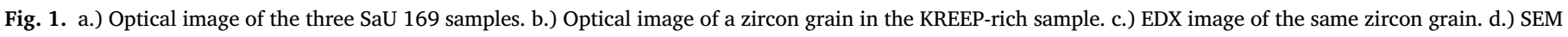
image of the zircon grain. 
during the EDX measurements. The presence of baddeleyite $\left(\mathrm{ZrO}_{2}\right)$ in the investigated area can be excluded because the mass spectra clearly show $\mathrm{Zr}$, Si, and $\mathrm{O}$ in the proper amounts for zircon $\left(\mathrm{ZrSiO}_{4}\right)$ (Fig. $2 \mathrm{~b}$ ). Typically, the zircons in the Sayh al Uhaymir 169 meteorite are $20-50 \mu \mathrm{m}$ large (Gnos et al., 2004; Lin et al., 2012), the one used for this study is almost $500 \mu \mathrm{m}$ in the long dimension, which is larger than the typical zircon grain in this meteorite. Panels b-d in Fig. 1 show the optical, EDX and scanning electron microscope (SEM) images of one zircon grain located on the KREEP-rich material of the sample.

\subsection{Mass spectrometric analysis of SaU 169}

\subsubsection{Relative sensitivity coefficients}

As with most analytic instruments performing quantitative analysis, the sensitivity of the laser ablation/ionization ion production may differ for each element and the physical and chemical properties of the sample materials, including light absorptivity, reflectivity, laser system, sublimation temperature, material porosity and chemical composition. Therefore, typically a calibration is necessary before the quantitative measurements of element abundances are to be conducted. These calibration factors, called relative sensitivity coefficients (RSCs), are defined as:

$R S C=$ measured element composition/real element composition

The RSC of an element $X$ is then calculated according to.Equation (1): RSC calculation

$R S C_{X}=\frac{\frac{1}{n_{X}}\left(\sum_{i} A_{i}^{X} / \sum_{i} A_{i}^{t o t}\right)}{\left(P_{X} / \sum_{i} P_{i}^{t o t}\right)}$,

where $n$ is the number of measured locations that contain the element $X$, $A_{i}^{X}$ is the peak area in the mass spectrum of the element $X$ for the $i$ th position, $A_{i}^{\text {tot }}$ is the total number of atoms of all the elements in the spectrum, $P_{X}$ the known atomic fraction of the element in the sample and $P_{i}^{\text {tot }}$ corresponds to the total fraction of all the measured elements (Neuland et al., 2014). The number of atoms of an element or isotope is obtained by the integration of the relevant mass peak area in the mass spectrum.

In addition, a sufficient number of measurement locations on the sample is needed to establish a statistically robust set of RSCs. With this
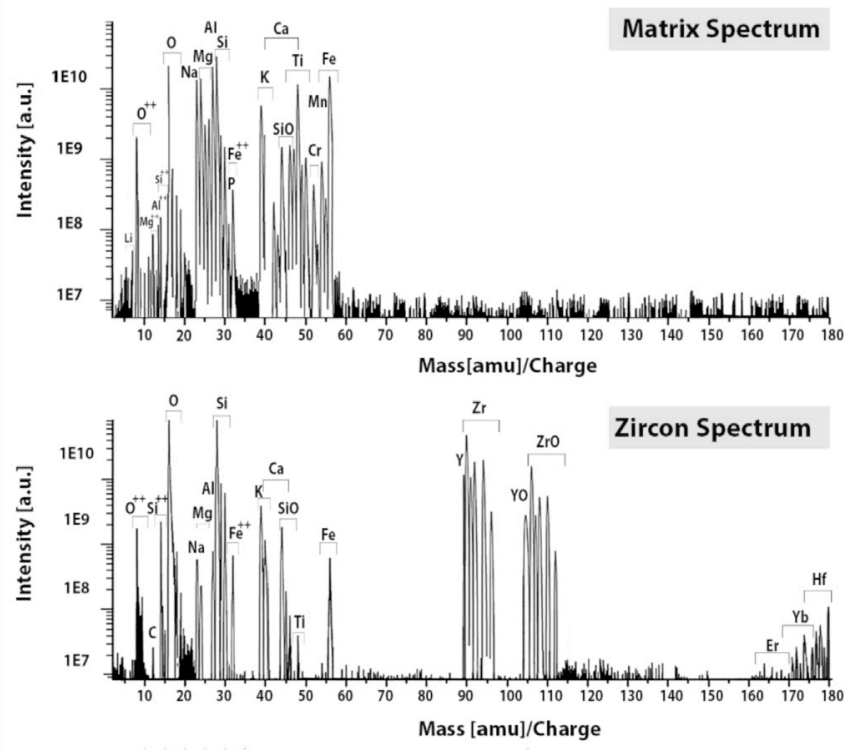

Fig. 2. Typical mass spectra for two positions on the KREEP-rich sample. Top panel: Position in the matrix area, bottom panel: measurement position inside the zircon grain. method, we determined such a robust set of RSCs by measurements on a sample of known composition and of similar texture and composition as the main sample. The material used to determine the RSC values should be similar in texture and composition to the sample to be investigated. For this study a set of RSCs for the main rock forming elements was determined on the regolith part of the SaU 169 meteorite, from which the bulk composition is known from previous studies (Gnos et al., 2004). Several locations in the most homogeneous areas were measured on the rock sample, where only small mineral grains were present.

In earlier investigations, comparisons of LMS with laboratory-sized instruments (ICP-CRI-MS and LA-ICPMS) showed good agreement with the LMS results (Grimaudo et al., 2017). Alternatively, a suitable standard of known composition should be used to establish the RSCs. Using standards for reference is certainly a viable option also for space applications. Moreover, duplication of the measurements in space by ones with the same instruments on the ground is often done to verify the interpretation of the measurements in space. Once the RSCs were determined and their accuracy tested on several mineral grains of the KREEP sample, the main measurements could be conducted.

\subsubsection{Elemental analysis and mineral identification}

Two typical mass spectra, one from the center of the zircon grain and one from the surrounding matrix area, are shown in Fig. 2. Mass spectra accumulated from a total number of $20^{\prime} 000$ laser shots at each position with a laser irradiance of $7.8 \mathrm{TW} / \mathrm{cm}^{2}$ were sufficient for accurate chemical analysis of the sample. The $20^{\prime} 000$ laser shots correspond to a depth of about a few tens of $\mu \mathrm{m}$.

The mass spectrum obtained from the fine-grained matrix area is shown in the top panel of Fig. 2. The spectrum shows that high abundances of $\mathrm{O}, \mathrm{Mg}, \mathrm{Al}, \mathrm{Si}, \mathrm{K}, \mathrm{Ca}, \mathrm{Ti}$ and $\mathrm{Fe}$, common in many rock-forming minerals, are readily detected. Abundances of $\mathrm{Li}, \mathrm{Na}$, and $\mathrm{K}$ may be affected by terrestrial contamination. However, a possible contamination would be restricted to the near-surface area, thus we excluded the first 500 laser shots from the analysis, i.e., starting the analysis about a $\mu \mathrm{m}$ below the surface. With a mass resolution of around $\mathrm{m} / \Delta \mathrm{m} \approx 350-400$ (FWHM) for most of the measured mass peaks, all the major elements can be measured with an accuracy of the isotopic ratios at the percent level. The mass spectrum from the interior parts of the zircon grain (bottom panel of Fig. 2) shows a distinct element composition. As expected the elements $\mathrm{Zr}, \mathrm{O}$ and $\mathrm{Si}$ are the most abundant elements in the spectrum, together with their oxides $\mathrm{ZrO}$ and $\mathrm{SiO}$ and some doubly charged ions $\mathrm{O}^{++}$and $\mathrm{Si}^{++}$, implying pure zircon is present. Hafnium and $\mathrm{Y}$ are common trace elements in zircon and can be measured at every position in the grain. The heavy REEs Yb, Dy, Gd, and Er can be detected at many positions in the grain. Also the light elements $\mathrm{Al}, \mathrm{Na}, \mathrm{K}$ and $\mathrm{Mg}$ as well as some Fe have been observed as trace elements in zircon before. The presence of these impurities in zircon agrees with earlier determinations of the chemical composition of the zircon grains in the Sayh al Uhaymir 169 meteorite [Lin et al., 2012].

Systematic measurements of the surface composition were done by investigating a grid of $15 \times 15$ locations, a total of 225 individual measurement spots. The laser probes an area of $\varnothing 15 \mu \mathrm{m}$. The distance between spots of $30 \mu \mathrm{m}$ was chosen to avoid any cross contamination of adjacent measurements. This gives a square area of $450 \mu \mathrm{m}^{2}$ on the KREEP-rich sample piece that was analyzed. The area was chosen to include parts of a zircon grain. Even though this raster pattern does not give a complete coverage of the investigated area the measurements can be plotted as $2 \mathrm{D}$ composition maps for each element identified in the mass spectra.

Plotting the element abundances on two-dimensional surface maps helps identifying regions with different element compositions. Fig. 3a represents the atomic fraction of the $\mathrm{Zr}$ abundances over the measured area. The map shows a very clear outline of the mineral grain with element abundances of around $15-16 \% \mathrm{Zr}$ atomic fraction for the analyses sampling only the grain. The abundance of $\mathrm{Ti}$ on the measured surface is shown in Fig. 3b. Positions with a Ti abundance of close to $20 \%$ 

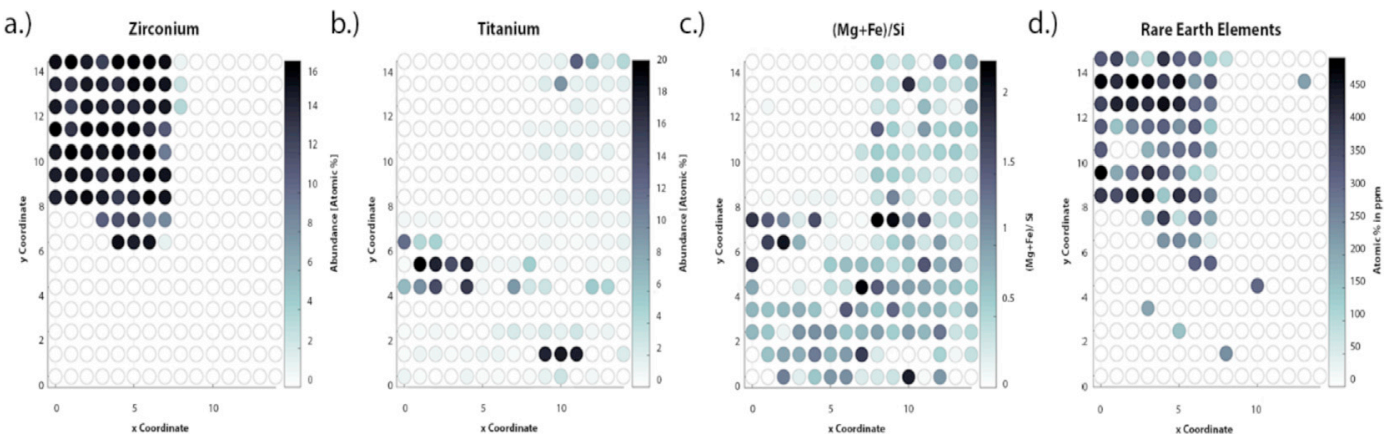

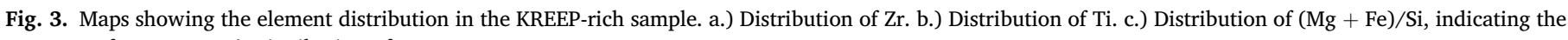
presence of pyroxene. c.) Distribution of REEs.

indicate the presence of ilmenite inclusions. The material surrounding the larger mineral grains consists of different fine-grained minerals. From the abundance of key elements these minerals can be identified. Fig. 3c shows that orthopyroxene, identified by $(\mathrm{Mg}+\mathrm{Fe}) / \mathrm{Si} \sim 1$, is very abundant in the matrix material. A surface map of the REE abundances is shown in Fig. 3d, with the most abundant REEs being $\mathrm{Yb}$ and Er in the zircon and La and Ce in the matrix material. It has to be noted that the map represents the total abundance of REEs, summing up the pertaining elements. The concentrations of the REEs outside the grain are highest in areas containing pyroxene. The light REE show highest abundances in the fine grained matrix material.

The chemical compositions of individual spot analyses are further investigated by their display in ternary diagrams shown in Fig. 4: one for the area on the KREEP part of the sample (Fig. 4a) and one for the spot analyses conducted on the regolith part of the sample (Fig. 4b). The elements and their ratios were chosen to differentiate between and to identify the most common minerals in lunar samples. The nominal element ratios of the components of the minerals correspond to the ideal chemical formula and are represented in the figures by the green square symbols, surrounded by a coloured area indicating a chemical variability of $\pm 10 \%$ for all three ratios, since the element compositions of minerals can vary to a certain degree in natural minerals due to substitution of different elements. Note that the uncertainties for the determination of the element ratios plotted in Fig. 4 are about 10\%, corresponding approximately to the size of the marker symbols. Spots measured inside the zircon grain are specially emphasized by red symbols. It is clearly visible that the majority of these positions have element ratios close to the nominal composition of zircon. Red spots outside this area correspond to the positions close to the rim of the zircon grain where other minerals are present in the spot sampled by the laser. The surrounding matrix material consists mainly of pyroxene (ortho- and clinopyroxene), plagioclase (anorthite-rich), K-feldspar as well as some ilmenite. Most of the measured positions in the matrix material consist of a mixture of several minerals as the grains in the matrix are smaller than the diameter of the laser spot $(\sim 15 \mu \mathrm{m})$. The data points for these analyses therefore lie between those of the end-member minerals.

Fig. $4 \mathrm{~b}$ shows an area of the matrix with compositions concentrated in the olivine/orthopyroxene area, thus with much higher homogeneity. Further analysis was done to determine the $\mathrm{Mg}+\mathrm{Fe}$ to $\mathrm{Si}$ ratio. A ratio near two is characteristic for olivine whereas a ratio near one corresponds to orthopyroxene. The area proved to be a large olivine grain with possible small inclusions of other minerals. The small drift towards $(\mathrm{Ca}+$ Ti) in Fig. $4 \mathrm{~b}$ is due to traces of Ti, present in various positions in the area, most likely due to small inclusions of ilmenite.

\subsection{Crystallization temperature of zircon}

Due to its unique chemical and physical durability zircon is commonly found in rocks other than the ones in which it originally crystallized. To place the mineral in a geological context it is crucial to identify the origin of zircon, especially since it is commonly used to perform radio-isotope dating to obtain time constraints on its formation. In addition, the trace elements in zircon preserve valuable information about its formation conditions. It has been found that the Ti concentration in zircon correlates with the temperature at which the grain originally crystallized. The log-linear dependence of the amount of Ti in zircon with its crystallization temperature was first proposed by Watson et al., in 2006 (Watson et al., 2006). At higher temperatures more Ti ions can substitute for either $\mathrm{Zr}$ or Si during crystallization. Once the temperature decreases, the Ti remains in the crystal structure.

In this study the abundance of Ti in one zircon grain was measured in 56 positions and an approximate crystallization temperature was derived from these measurements. The estimate is calculated using the revised dependence of the Ti abundance, $\log (\mathrm{Ti})$, on the crystallization temperature $T$ according to Ferry and Watson (2007):

Equation (2): Titanium-temperature dependence
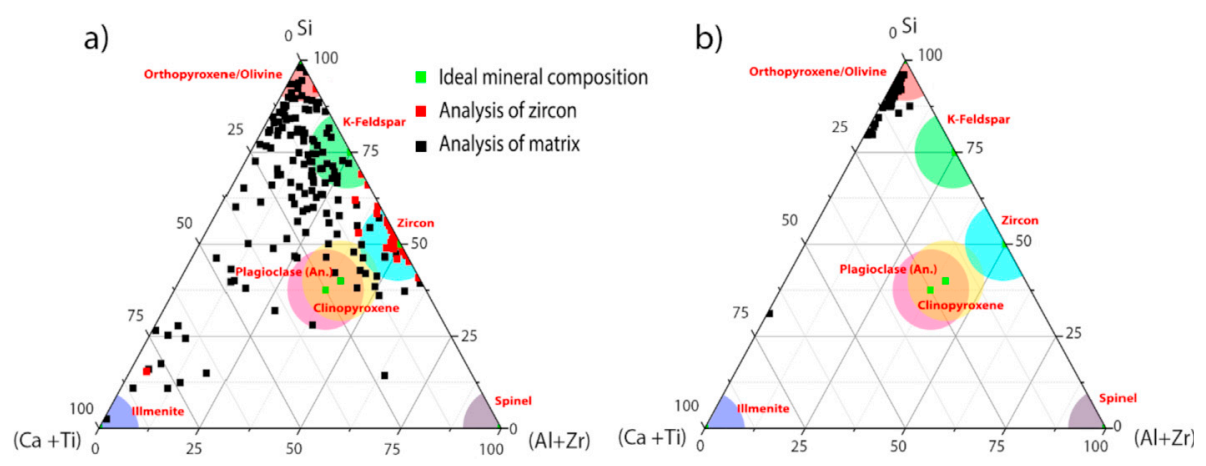

Fig. 4. Ternary diagrams to visualize the minerals present in the sample: a) Area containing a zircon grain on the KREEP-rich sample; b) Area on the border between the KREEP-rich and regolith parts of the meteorite. 
$\log (T i)=5.711-\frac{4800}{T(K)}-\log \left(a_{\mathrm{SiO}_{2}}\right)+\log \left(a_{\mathrm{TiO}_{2}}\right)+\log \left(a_{\mathrm{ZrSiO}_{4}}\right)$.

The two values $a_{\mathrm{SiO}_{2}}$ and $a_{\mathrm{TiO}_{2}}$ are concentrations corrected for reduced oxide activities (chemical potential) of $\mathrm{Si}$ - and Ti-oxide. They depend on the composition of the parent magma during crystallization. The exact composition of the melt from which the zircon crystallized is not known, but a common $\mathrm{SiO}_{2}$ content of lunar basalts from which zircon can crystallize is $50-60 \% \mathrm{wt} \%$. Lunar basalts tend to be saturated in ilmenite and not rutile, thus the activity of $\mathrm{TiO}_{2}$ is lower than unity. In a silicate melt $a_{\mathrm{TiO}_{2}}$ is typically 0.6-0.9 (Watson et al., 2006; Ferry and Watson, 2007).

The $a_{\mathrm{ZrSiO} 4}$ takes into account the incorporation of other trace elements in the zircon grain. Using Raoult's law it follows that

$a_{\mathrm{ZrSiO}_{4}}=X_{\mathrm{ZrSiO}_{4}}$

where $X_{\mathrm{ZrSiO} 4}$ is the mole fraction of $\mathrm{ZrSiO}_{4}$ in the grain. To minimize possible contributions from nearby minerals during analysis, only positions clearly separated from the grain boundary were used. These analyses were selected by their molecular fraction of $\mathrm{ZrSiO}_{4}$ being no less than $95 \%$, thus $a_{\mathrm{ZrSiO} 4}$ is close to 1 . The element abundances were calibrated using the same RSCs determined earlier for the mineralogical analyses. Using these values, the calculated temperature will likely be a slight underestimation of the real crystallization temperature. The results obtained with the Ti-in-zircon thermometer are visualized in a histogram plot shown in Fig. 5. The temperatures range from 850 to $1450 \mathrm{~K}$, with an average temperature of $1176 \mathrm{~K}$ and a variance of $140 \mathrm{~K}$. The Ti content varied in the measured positions without showing a consistent pattern. The thermometer tends to yield a minimum crystallization temperature because the maximum $\mathrm{Ti}$ content is only achieved if sufficient $\mathrm{Ti}$ is available for partitioning into zircon. Thus, lower estimated temperatures can indicate a reduced activity of $\mathrm{Ti}$, that is common if no Ti-phase is present. However, the KREEP basalt contains ilmenite, minimizing this problem since it assures constant activity of $\mathrm{Ti}$ in the mineral assemblage. Consequently, the most likely cause for the range in temperatures obtained is crystallization of zircon during magma cooling and solidification.

The estimated high temperatures are very close to the melting temperature of KREEP-rich basaltic melts. Basaltic melts have a temperature of around $1200{ }^{\circ} \mathrm{C}$ during eruption. It is therefore reasonable to assume that the analyzed grain could have formed in a slowly cooling and evolving melt when it reached zircon saturation.

\subsection{Measurements of trace elements with the use of a high-voltage pulser}

In the last measurement campaign, the ion optics of the LMS instrument was coupled with a high-voltage pulser. The pulser generates a temporary pulse of variable duration on one electrode of the ion-optical

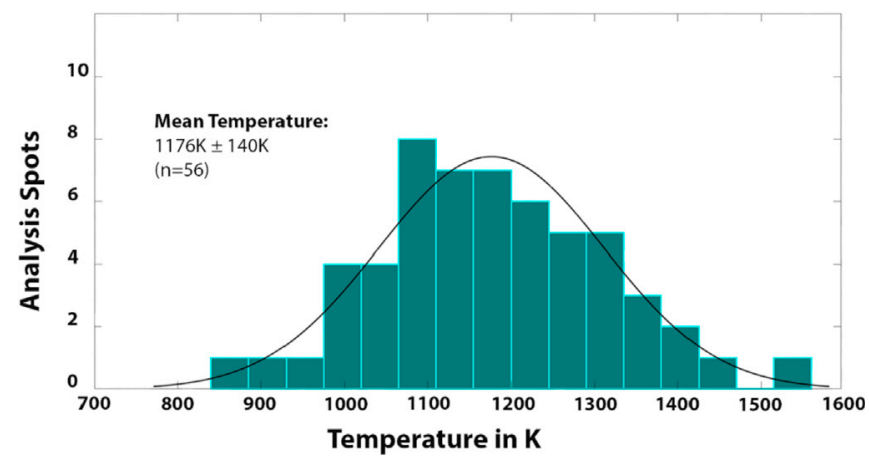

Fig. 5. Histogram of the crystallization temperature derived from the Ti content in a zircon grain. The determined temperature follows a normal distribution with $\mu=1176 \pm 140 \mathrm{~K}$. system, which prevents the ions from reaching the MCP detector. With this set-up, the high-voltage pulse duration can be chosen in such a way that the light, high abundant elements are prevented from reaching the detector system. This leads to an increased detection sensitivity of the heavy elements due to the increased number of available and fresh pores for signal amplification of the MCPs of the detector system (Wiesendanger et al., 2017).

A set of measurements was then conducted on the KREEP material of SaU169 using the high-voltage pulser. The pulse duration was adjusted to detect only elements with masses higher than Fe. Several positions, both in and around zircon grains were analyzed. It was found that the SNR of the measured REEs could be increased by a factor of four while comparing to the previous measurement campaign. This allowed for resolving of the individual mass peaks. Additionally, ${ }^{238} \mathrm{U}$ and ${ }^{232} \mathrm{Th}$, which have abundances in the ppm range, were readily detected on several positions in the zircon. Radiogenic $\mathrm{Pb}$, from the decay of $\mathrm{U}$ and $\mathrm{Th}$ accumulated in the zircon, was only detected at one location. Even though a single measurement cannot be statistically significant, a rough age estimation of the grain was determined from these data using the ratio of the measured ${ }^{238} \mathrm{U}$ and ${ }^{206} \mathrm{~Pb}$ peaks according to (Allègre, 2008):

Equation (3) Estimation of the zircon crystallization age

$t=\frac{1}{\lambda_{238}} \ln \left(\frac{P b}{U}+1\right)$.

The RSC values for $\mathrm{Pb}$ and $\mathrm{U}$ could not be determined in this measurement. Using a correlation that was found between the RSCs and the first ionization potential of an element (Neuland et al., 2016; Zhang et al., 2013) one can obtain an estimation of the RSC values of Pb and U. Using these RSCs, we determined the crystallization age of $t=(3517 \pm 130)$ $-10^{6}$ a. This age is slightly lower than earlier age determinations with laboratory instruments. From the ${ }^{207} \mathrm{~Pb} /{ }^{206} \mathrm{~Pb}$ isotope measurements Gnos et al. (2004) derived an average age of $t=(3909 \pm 13) \bullet 10^{6}$ a from measurements of 12 zircon grains, and Lin et al. (2012) derived an average age of $t=(3921 \pm 3) \bullet 10^{6}$ a from 5 zircon grains. From U-Pb measurements Liu et al. (2009) determined an age $t=(3918 \pm 9) \bullet 10^{6} \mathrm{a}$ from 9 analyses on 4 zircon grains. Since the sample is brecciated, there is the possibility that the large zircon grain is derived from an external source and thus the small grains do not record the same age as the large grain. More measurements at different locations on the sample will be needed to improve the statistical quality of the age determination in future measurements with the LMS. Moreover, ${ }^{207} \mathrm{~Pb} /{ }^{206} \mathrm{~Pb}$ should be preferred for radio-isotope dating, as the ratio between the different $\mathrm{Pb}$ isotopes are independent of RSC values. Nevertheless, the current measurement demonstrated that using LMS it is possible to conduct relatively accurate in-situ radio-isotope age determinations on single minerals in a rock on a planetary surface. Moreover, these in-situ measurements are valuable to pick suitable samples on a planetary surface for their transport to Earth within a sample return mission.

\section{Conclusion and outlook}

The lunar meteorite Sayh al Uhaymir 169, that contains KREEP rich material, was investigated with our miniature laser ablation time-offlight mass spectrometer to simulate the expected performance of insitu measurements on the surface of a planetary body. The abundances of the major elements on the KREEP part of the rock were measured and plotted on a two-dimensional surface map to identify different mineral phases in the sample. The element distribution maps reveal the grain boundaries and allow identification of individual minerals and their major and trace element compositions.

In the second measurement campaign the instrument was coupled with a HV pulser to increase sensitivity of the measurements. It could be shown that the detection sensitivity for heavy trace elements can be significantly improved while removing light elements from the analyzed ion beam. This study provides also measurements of $\mathrm{U}$, Th and $\mathrm{Pb}$ 
elements present inside a single zircon grain. With the abundances of $U$ and $\mathrm{Pb}$ in the zircon grain a preliminary age estimation of the zircon could be made and was found to be in reasonable agreement with ages reported in literature. Moreover, from the Ti concentration in the zircon grain a crystallization temperature was derived. Thus, by employing such an instrument on a planetary surface, scientific useful in-situ measurements on the element composition, the mineralogy, petrology and dating of surface samples can be accomplished.

\section{Acknowledgement}

We gratefully acknowledge the financial support by the Swiss National Science Foundation.

\section{References}

Allègre, C.J., 2008. Isotope Geology, first ed. Cambridge University Press, Cambridge. Bieler, A., et al., 2011. Optimization of mass spectrometers using the adaptive particle swarm algorithm. J. Mass Spectrom. 46, 1143-1151.

Ferry, J.M., Watson, E.B., 2007. New thermodynamic models and revised calibrations for the Ti-in-zircon and Zr-in-rutile thermometers. Contrib. Mineral. Petrol. 154, 429-437.

Grimaudo, V., et al., 2015. High-resolution chemical depth profiling of solid material using a miniature laser ablation/ionization mass spectrometer. Anal. Chem. 87, 2037-2041. https://doi.org/10.1021/ac504403j.

Grimaudo, V., et al., 2017. 3D chemical imaging of ternary Cu-Sn-Pb alloys using Femtosecond Laser Ablation/Ionization Mass Spectrometry. Anal. Chem. 89 (3), 1632-1641. https://doi.org/10.1021/acsanalchem.6b03738.

Gnos, E., et al., 2004. Pinpointing the source of a lunar meteorite: implications for the evolution of the moon. Science Magazine 305, 657-659.

Hays, L.E., et al., 2017. Biosignature preservation and detection in Mars analog environments. Astrobiology 17 (4), 363-376.

Liu, D., et al., 2009. Age of zircons in the impact-melt breccia in SaU 169 lunar meteorite: Beijing SHRIMP II study. Lunar Planet. Sci. Conf. 40. \#2499 (abstr.).

Lin, Y., et al., 2012. Very high-K KREEP-rich clasts in the impact melt breccia of the lunar meteorite SaU 169: new constraints on the last residue of the lunar magma ocean. Geochem. Cosmochim. Acta 85, 19-40.

Maurice, S., et al., 2012. The ChemCam instrument suite on the Mars science laboratory (MSL) rover: science objectives and mast unit description. Space Sci. Rev. 170, 95-166. https://doi.org/10.1007/s11214-012-9912-2.

Meyer, S., et al., 2017. Fully automatic and precise data analysis developed for time-offlight mass spectrometry. J. Mass Spectrom. 52, 580-590.
Neubeck, A., et al., 2016. Mineralogical determination in situ of a highly heterogeneous material using a miniaturized laser ablation mass spectrometer with high spatial resolution. Int. J. Astrobiol. 15, 133-146.

Neuland, M.B., et al., 2014. Probing the Allende meteorite with a miniature laser-ablation mass analyzer for space application. Planet. Space Sci. 101, 196-209.

Neuland, M., et al., 2016. Quantitative measurement of the chemical composition of geological standards with a miniature laser ablation/ionization mass spectrometer designed for in situ application in space research. Meas. Sci. Technol. 27 article ID 035904.

Rieder, R., et al., 1997. The chemical composition of martian soil and rocks returned by the mobile alpha Proton X-ray spectrometer: preliminary results from the X-ray mode. Science 278, 1771-1774.

Rieder, R., et al., 2003. The new Athena alpha particle X-ray spectrometer for the Mars Exploration Rovers. J. Geophys. Res. 108 (E12), 8066. https://doi.org/10.1029/ 2003JE002150.

Riedo, A., et al., 2013a. Highly accurate isotope composition measurements by a miniature laser ablation mass spectrometer designed for in situ investigations on planetary surfaces. Planet. Space Sci. 87, 1-13.

Riedo, A., et al., 2013b. Coupling of LMS with a fs-laser ablation ion source: elemental and isotope composition measurements. J. Anal. Atomic Spectrom. 28, 1256-1269 (Riedo et al., 2013b).

Riedo, A., et al., 2017. High-speed microstrip multi-anode multichannel plate detector system. Rev. Sci. Instrum. 88, 045114.

Rohner, U., Whitby, J., Wurz, P., 2003. A miniature laser ablation time-of-flight mass spectrometer for in situ planetary exploration. Meas. Sci. Technol. 14, 2159-2164.

Russ, J.C., 1984. Fundamentals of Energy Dispersive X-Ray Analysis, sixth ed. Butterworths Monographs in Materials, London.

Russel, S., et al., 2003. The meteoritical Bulletin, No.87. Meteorit. Planet. Sci. 38 (Nr.7), A189-A248.

Tolstikhin, I.N., Kramers, J.D., 2008. The Evolution of Matter: from the Big Bang to the Present Day Earth, first ed. Cambridge University Press, Cambridge.

Tulej, M., et al., 2015. Chemical Composition of Micrometer-Sized Filaments in an Aragonite Host by a miniature laser ablation/ionization mass spectrometer. Astrobiology 15, 669-682.

Watson, E.B., Wark, D.A., Thomas, J.B., 2006. Crystallization thermometers for zircon and rutile. Contrib. Mineral. Petrol. 151, 413-433.

Wiens, R.C., et al., 2012. Sci. Rev., the ChemCam instrument suite on the Mars. Science Laboratory (MSL) Rover: Body Unit and Combined System Tests 170 (1-4), 167-227, 2012.

Wiesendanger, R., et al., 2017. Improved detection sensitivity for heavy trace elements using a miniature laser ablation ionization mass spectrometer. J. Anal. Atomic Spectrom. 32, 2182-2188.

Wiesendanger, R., et al., 2018. Chemical and optical identification of micrometer sized 1.9 billion-year-old fossils by combining a miniature LIMS system with an optical microscope. Astrobiology 18 (8), 1071-1080 (accepted).

Zhang, B., et al., 2013. Minimizing matrix effect by femtosecond laser ablation and ionization in elemental determination. Anal. Chem. 86, 4507-4511. 